\title{
ESTRATEGIAS DE ENSEÑANZA PARA EL ACOMPAÑAMIENTO DE LAS TRAYECTORIAS DE LOS ESTUDIANTES DEL TRABAJO FINAL DE CARRERA DE LA FAU-UNNE
}

Sandra R. FOGAR

sanfogar@gigared.com

Jefa de Trabajos Prácticos de Trabajo Final de Carrera, FAU-UNNE.

Palabras Clave: Trayectorias estudiantiles; estrategias; prácticas docentes. Keywords: student trajectories; strategies; teaching practices.

\section{RESUMEN}

Se presentan resultados de la indagación teórica sobre trayectorias de los estudiantes, como noción que posibilita analizar la enseñanza y el aprendizaje en clave social. La perspectiva de las trayectorias inscribe el estudio de las estrategias de enseñanza en una visión que disloca la concepción tradicional del rol docente e invita a mirar la enseñanza como práctica que involucra incertezas docentes y capacidad de acompañar a los estudiantes hacia procesos de emancipación con respecto a los mecanismos de sujeción que obturan el conocimiento. Se enuncian, pues, interrogantes sobre la enseñanza en el TD, que surgen de las propias prácticas.

\begin{abstract}
Results of theoretical inquiry into trajectories of students are presented here as a notion that allows to analyze teaching and learning in a social key. The perspective of the trajectory inscribes the studying of teaching strategies in a vision that dislocates the traditional conception of the teacher's role and invites to look at teaching as a practice that involves teacher's uncertainties and the ability to accompany students to the processes of emancipation respect to the mechanisms, which blocks the knowledge. Therefore, they are set out questions about teaching in the TD, arising from their own practices.
\end{abstract}




\section{OBJETIVOS}

- Indagar el estudio de las trayectorias en Argentina. - Analizar algunos aspectos de las prácticas docentes desde la perspectiva del estudio de trayectorias.

\section{INTRODUCCIÓN O PLANTEO DEL PROBLEMA}

“... ¿Si yo tuviese que enseñarles arquitectura? Es una pregunta bastante embarazosa..."

LE CORBUSIER. Architectural Design, Vol. 29, febrero de 1959

El presente trabajo se inscribe en la investigación en curso Aportes para la elaboración de estrategias didácticas para la integración de conocimientos del Proceso de Diseño Proyectual en el Trabajo Final de Carrera de de la FAU-UNNE', desarrollada en el marco del PROMARQ y acreditada por la Secretaría General de Ciencia y Técnica de la Universidad Nacional del Nordeste.

El análisis de la práctica del diseño proyectual en los talleres de la FAU-UNNE puso en evidencia la influencia de los recorridos formativos de los estudiantes y el papel de las instituciones educativas en los procesos de formación. Desde esta perspectiva, la cuestión de la enseñanza resulta en un problema cuya complejidad exige un abordaje que trasciende tanto lo pedagógico-didáctico como lo institucional, en sentido restringido, pues es solo desde un análisis social que puede comprenderse cómo se construye el desempeño de estudiantes y profesores. Con ello se reconoce que el estudio de las trayectorias puede conducir de la reflexión sobre los estudiantes a la reflexión sobre las prácticas educativas en la universidad, en la medida en que esta no es impermeable a los procesos históricos que inciden en la constitución de las subjetividades sociales y determinan también lógicas y recorridos institucionales. El estudio de las trayectorias de los estudiantes posibilita, de esta forma, analizar la enseñanza y el aprendizaje, en clave social.

Los estudios sobre trayectorias, relativamente nuevos en la Argentina, tensionan las prácticas docentes inscritas en el conjunto de prácticas institucionales, al poner en valor los procesos sociales que tienen lugar en las instituciones y que incluyen los procesos y estrategias de transmisión y construcción de conocimientos. Asimismo, se reconocen las relaciones entre esos procesos y la construcción del desempeño profesional de los estudiantes, en el que, sin embargo, no tienen menos incidencia las experiencias sociales de cada estudiante como sujeto individual y social, o sea, aquellas experiencias vividas antes de su ingreso en la universidad e incluso fuera de algún ámbito educativo.

La tradición en educación pone el peso de los problemas en el aprendizaje, con lo que los estudiantes terminan siendo depositarios de la responsabilidad en el éxito o el fracaso alcanzados. Sin duda, al dominio de esta concepción en el ámbito educativo ha contribuido el estatus alcanzado por la Psicología en el siglo XX, así como el abuso que hizo la escuela de presupuestos provenientes de esa disciplina. Por supuesto, esta no es la única causa del primado de esa concepción en las prácticas educativas. Hay una razón de mayor peso para haber evitado la consideración de lo social en los procesos educativos: el dominio del liberalismo, como concepción ideológica que pone en el centro de la escena al individuo. Es por ello que si bien lo social nunca quedó fuera del discurso pedagógico, gravitó, sin embargo, como problema que resolver desde una lógica del orden que concibe educación para la adaptación y supone la superioridad de 
"La tradición en educación pone el peso de los problemas en el aprendizaje, con lo que los estudiantes terminan siendo depositarios de la responsabilidad en el éxito o el fracaso alcanzados."

los conocimientos académicos con respecto a los saberes populares y a los conocimientos adquiridos a través de experiencias no escolares o experiencias educativas no formales. Todo ello en estrecha relación con la función asignada por el sistema capitalista a la educación, reducida a la selección de los individuos capaces de ocupar los puestos de trabajo, según las demandas del mercado.

Esa tradición ubica al docente en una posición poco problemática, más cómoda y segura. Lo coloca en un lugar de portador de un conocimiento considerado universal y superior, que es necesario transmitir a las nuevas generaciones para "ilustrarlas". Para cumplir el mandato de ilustrar a niños y jóvenes, alcanza con que el docente posea cierto dominio del conocimiento científico que enseñar y, sobre todo, sepa cómo hacerlo, en el sentido de que domine ciertas técnicas de transmisión.

La perspectiva de las trayectorias de los estudiantes disloca la posición tradicional dominante en la docencia, que naturaliza el lugar del profesor como ejecutor de un rol, como plantea SOUTHWELL (s/f: 3), ajustado a modelos que ataron la certeza de lo instrumental a una relación autoritaria y empobrecida con el conocimiento. En esa concepción no hay lugar para la incerteza, considerada improductiva. Ello conduce inevitablemente a un posicionamiento docente en el cual no cabe la pregunta, requisito indispensable para el logro de la autonomía. Dado que la cuestión de la autonomía profesional corresponde al orden de la subjetividad, esta se consigue apuntando la reflexión sobre las prácticas en el análisis de la historia personal del docente, inscrita en la historia institucional y social. La reflexión sobre las prácticas involucra, pues, la institución -o mejor, las instituciones - en que estas se configuran, se desarrollan y se legitiman. Los estudios de trayectorias vienen a poner en valor aquello que quedó fuera de la tradición en educación, tanto con respecto a los estudiantes como con respecto a los docentes.

\section{DESARROLLO}

Como plantean FOGAR, M. Y SILVA, C. (2013): "Diversos estudios (TERIGI, 2007; GLUTZ, 2006; KAPLAN Y FAINSOD, 2006; FOGAR Y OTROS, 2010) muestran que las trayectorias formativas de los estudiantes se construyen en las relaciones entre competencias personales, condiciones institucionales, logros académicos, calificaciones obtenidas y respuestas institucionales a las necesidades socioeducativas. A partir de las experiencias individuales y colectivas vividas en esas trayectorias, se construye el desempeño..., en su complejidad"2. Considerada en sentido amplio, la noción de trayectoria escolar permite reconstruir la historia personal, escolar y social de los estudiantes. KAPLAN Y FAINSOD (2006) la definen como el recorrido que realiza el alumno por grados, ciclos y niveles a lo largo de su historia escolar. Este recorrido incluye el registro de los obstáculos en las biografías personales de los estudiantes, así como sus decisiones acerca de interrupciones y "desvíos" en esos recorridos. (FOGAR, M. Y SILVA, C., 2013: 7).

Para NICASTRO Y GRECO (2012) la trayectoria es un recorrido, un camino en permanente construcción $y$, por tanto, tal como señala ARDOINO (2005), no modelizable, no totalmente anticipable y no susceptible de concreción a través de pasos mecánicamente establecidos, según pautas o regulaciones internas o externas. La trayectoria

2 FOGAR, MARIELA DEL CARMEN Y SILVA, CARLOS ALBERTO (2014). Los estudiantes y su formación. Escenarios y tensiones en la educación técnica. Estudio de casos del Profesorado en Tecnología del INTET y P. Revista Theomai. Estudios Críticos sobre Sociedad y Desarrollo. N. ${ }^{\circ}$ 30, Conflictos y Problemáticas Sociales, segundo semestre de 2014. ISSN: 1515-6443, pp. 172-180. 
es más bien "itinerario en situación". La idea de trayectoria como camino admite la interrupción, el atajo, el desvío, los otros tiempos. La trayectoria implica sujetos en situación de acompañamiento. Esa situación requiere una mirada oblicua o, en el sentido de CHALMERS (1995), una mirada de ruptura epistemológica, de quiebre con lo instituido, con lo naturalizado, con los guiones académicos. Se trata de la mirada dislocada, postulada por MASSCHELEIN (2006), quien invita a "Dislocar la propia mirada de modo de poder ver diferente, ver lo visible (en tanto 'las distancias, calveros, y perspectivas' no es que están escondidas ni están en algún 'más allá') y ser transformados... Eso es caminar: dislocamiento de la mirada que permite la experiencia, no solo un transcurrir pasivo (estar bajo el mando de otro) sino un modo de abrirnos paso en el camino" (MASSCHELEIN, J., 2006: 2).

Desde esta perspectiva, no se trata de modelar la trayectoria de un estudiante, lo que implicaría acompañar la sumatoria de pasos establecidos dentro del sistema académico, según pautas y resultados prescritos para todos, en un currículum acorde con la estructura del sistema. Esto es así porque, desde la lógica modélica, en el acompañamiento del recorrido no importa quién es el estudiante, concebido desde un perfil tipificado, la mayoría de las veces según un "deber ser" o un imaginario en el que el deber ser social se conjuga con proyecciones de los docentes, de la imagen que tienen de sí mismos como estudiantes o como profesionales, representada como ideal universalizable.

Lo mismo ocurre, por supuesto, con el otro componente de la relación educativa: el docente, valorado según un currículum personal concebido como un conjunto de certificaciones que acreditan idoneidad para un puesto de trabajo. Docentes y estudiantes resultan, desde la concepción tradicional, puestos como anónimos, y el ámbito de la relación entre ambos resulta invisibilizado.
En el estudio de las trayectorias prima la pregunta sobre quién o quiénes son los sujetos involucrados en la situación educativa y el complejo entramado que, en su configuración, posibilita el despliegue de un sinnúmero de fenómenos propios de la institución del sujeto y del colectivo, al decir de CASTORIADIS (1989), como instituciones primeras. Esas instituciones primeras que se expresan en las instituciones segundas, familia, escuela, etc., y son el requisito de funcionamiento de lo social. De aquí que el requisito para leer las trayectorias de los estudiantes sea el diálogo con ellos, para que surja la narración. No se trata aquí de diálogo en el sentido restringido del hablar con, sino de una forma de comunicación que exige, por parte del docente, una formación tal que posibilite ver más allá de lo dicho, lo mostrado, en definitiva, lo aparente, para avanzar en un proyecto de formación de un colectivo de estudiantes, inscrito en un currículum abierto y susceptible de reformulación, a partir del diálogo con lo real.

Lo antedicho permite reconocer la vinculación de la perspectiva de las trayectorias con los postulados pedagógicos fundamentales de la psicosociología francesa, que tiene a FERRY como uno de sus mayores exponentes. La formación, en el sentido de FERRY, involucra no solo el conocimiento del aparato conceptual propio de la o las disciplinas de un campo, sino sobre todo la capacidad de adaptación y de ruptura con las adaptaciones requeridas por el sistema.

Por supuesto, los procesos de socialización no son independientes de los procesos intelectuales. Efectivamente, la relación entre el pensar el mundo y construirlo es objeto de la filosofía desde los inicios mismos de la Modernidad. Por otra parte, los conceptos piagetianos de asimilación y acomodación resultan sustanciales para comprender la relación entre construcción social del conocimiento y construcción de la subjetividad colectiva. 
"Los relatos de los estudiantes hablan de sus condiciones familiares y sociales, sus condiciones académicas en el momento de la cursada, sus valoraciones acerca de los conocimientos científicos adquiridos y de la práctica profesional..."

Aunque pueda parecer redundante, tiene sentido recalcar que en un abordaje de las trayectorias trata de las experiencias en sentido no restringido al conocimiento académico. Por ello, resulta valioso preguntarse ¿a qué experiencias académicas y no académicas remiten los relatos de los estudiantes sobre sus aprendizajes? Este interrogante obliga a mirar en qué medida los relatos de los estudiantes sobre el aprendizaje de contenidos académicos remite claramente a la relación del aprendizaje con la construcción de estrategias institucionales y personales y la selección, la reproducción o la resistencia de estos en el empleo de aquellas, hasta la construcción de su desempeño, primero como estudiantes y luego como profesionales.

Los relatos de los estudiantes ${ }^{3}$ hablan de sus condiciones familiares y sociales, sus condiciones académicas en el momento de la cursada, sus valoraciones acerca de los conocimientos científicos adquiridos y de la práctica profesional, etc. Desde su consideración, sin más, se podría caer nuevamente en el error de pensar tanto que existen condiciones ideales para el aprendizaje como que, para ser buenos enseñantes, es necesario ser capaces de advertir las dificultades o carencias de los estudiantes. La reflexión sobre las trayectorias conduce a otro tipo de lectura, que posibilita advertir el sentido social atribuido al aprendizaje y a la experiencia universitaria. A la vez, esto abre las puertas a la reflexión sobre el sentido social de las prácticas docentes en la universidad.

Dado el carácter de indagación teórica de este trabajo, no se analiza cada uno de los elementos de los relatos, solo se consideran algunos, que pueden servir como ejemplos del abordaje de un estudio sobre las trayectorias de los estudiantes. Según la

\footnotetext{
3 Para la recopilación de la información se utilizó la encuesta estructurada, que se aplicó al total de inscritos en la asignatura TFC, de la UP "A", al comienzo del dictado de los años académicos 2014-2015, y se entrevistó a quince mujeres y quince varones de entre veinticuatro y treinta años.
}

información relevada, los estudiantes pertenecen a sectores socioeconómicos diversos y tienen experiencias de formación también diferentes, tanto en cuanto a las trayectorias escolares como a la formación desde el ingreso en la universidad. Alrededor del $70 \%$ de los estudiantes costea los estudios a través del empleo, en la administración pública, en empresas constructoras, en comercios relacionados con la industria de la construcción o de la gastronomía, en el desempeño docente en escuelas técnicas o como profesores particulares, o como técnicos de PC. Aproximadamente entre el 0,5 y el $1 \%$ de las estudiantes mujeres lo hacen a través del empleo doméstico o como empleadas de limpieza en alguna empresa. Un tercio de los estudiantes trabajadores son madres/padres y sostén de familia. De entre ellos, el 50 \% son madres solteras. Una pequeña minoría se asume como militante de una agrupación estudiantil. Aproximadamente la mitad de los estudiantes utiliza el transporte público urbano o interurbano para movilizarse a la universidad. Los demás se trasladan en bicicleta, motocicleta o auto propios.

Con respecto a la experiencia de aprendizaje, la mayoría expresa que prefiere "los trabajos teóricos" antes que encarar "una propuesta de diseño". Esta apreciación contrasta con las aspiraciones de los mismos estudiantes, ninguno de los cuales ha hecho una opción por la investigación. Pero mayor aún es la contradicción con respecto a las prácticas de indagación que utilizan. La experiencia de enseñanza en la cátedra pone en evidencia que la mayoría se vale de imágenes recuperadas de sitios de Internet y revistas de Arquitectura como herramientas fundamentales para la práctica del diseño, que luego reproduce sin más y con criterios arbitrarios. Estas prácticas hablan, sin duda, de la ausencia de rigor científico en la práctica del diseño arquitectónico, lo que es claramente opuesto a una actitud teórica que creen asumir y debería conducir más claramente a una actitud investigativa como la que se espera alcancen a través de la experiencia 
académica. No alcanza, sin embargo, con pensar este fenómeno restringiéndolo a los estudiantes y al ámbito académico, pues los relatos evidencian que tanto esas creencias como esas actitudes fueron construidas mucho antes de la experiencia universitaria, es decir, en las experiencias sociales marcadas por el mercado y las prácticas de consumo que este impone y alcanzan incluso al conocimiento científico.

Con respecto al significado que atribuyen a la experiencia estudiantil en la universidad y al modo en que se representan su futura inserción como arquitectos en el mercado laboral, resulta significativo mencionar que, a pesar de que ya transitan por el mundo del trabajo, la mayoría expresa que la idea de "encontrarse solo en el ejercicio profesional" le produce temor, sentimiento que atribuyen a la "falta de conocimiento, so-bre todo de las materias tecnológicas". Estas expresiones hablan de las dificultades que encierra el "salto" de la vida estudiantil a la vida profesional, cuestión muchas veces naturalizada como "simple paso" de una actividad a otra por parte de docentes con una vasta trayectoria en el ejercicio de la profesión y que se han incorporado a un mercado laboral muy diferente del actual — hoy más competitivo en términos de la existencia de gran cantidad de recursos humanos, algunos con amplias acreditaciones- Al respecto podría considerarse también que la experiencia social de la década del 90, en que el contexto neoliberal significó, entre otras cosas, el achicamiento del mercado laboral y la precarización del trabajo, sigue presente como un fantasma, sobre todo para los sectores subalternos que experimentaron directamente los efectos del modelo exclusor. Esto explica además el interés de los estudiantes por desarrollar trabajos de diseño en el marco de convenios de trabajo entre la Facultad de Arquitectura e instituciones de la administración pública, lo que suponen les asegurará un puesto de trabajo inmediatamente después de la finalización de la carrera.
En relación con las estudiantes mujeres, casi la totalidad dice tener serias dificultades para apropiarse de saberes propios de las ciencias tecnológicas y técnicas constructivas y, por el contrario, poseer habilidades para las ciencias humanas. Este discurso muestra que las representaciones que las estudiantes tienen sobre sí mismas coinciden con el discurso social dominante que adjudica a varones y mujeres capacidades diferenciadas, y reserva para los primeros la capacidad de apropiarse de conocimientos provenientes de las llamadas tradicionalmente "ciencias duras", a las que a la vez se les atribuye un estatus superior. De hecho, diversos estudios sobre la cuestión de las representaciones dan cuenta de que lo antedicho no es un fenómeno exclusivo de la universidad, sino que se produce en todos los niveles del sistema educativo y constituye uno de los tantos mecanismos de reproducción social.

Si bien la relación entre las representaciones y temores de los estudiantes con la enseñanza en la universidad no es lineal, el empleo de estrategias que promueven el trabajo autónomo y la reflexión sobre lo social podría contribuir a reforzar la confianza en sus propias capacidades, así como a quebrar la creencia de que mayores conocimientos de las materias tecnológicas garantizarían un desempeño exitoso. Esa creencia impide reconocer que es el conjunto de saberes y conocimientos teóricos, operando como andamiaje conceptual, la única garantía del saber hacer, tanto en situaciones previsibles como en aquellas inesperadas. En esta línea de pensamiento, las acciones de extensión universitaria en el marco de convenios con instituciones públicas tienen un plus de valor, aunque ello no pueda asegurar el empleo.

Más que una respuesta inmediata, parece adecuado formularnos algunos interrogantes acerca de las prácticas docentes: ¿qué estrategias de enseñanza se utilizan?; ¿en qué medida esas estrategias posibilitan articular el campo del 
"Es precisamente a esa decisión de vivir juntos la que puede y debe contribuir la arquitectura, concebida y ejercida como profesión cuya razón de ser es lo social, en el sentido de que esta viene a resolver espacios para el desarrollo de actividades colectivas..."

conocimiento científico con el ejercicio de la profesión?; ¿cuáles estrategias resultan adecuadas para problematizar lo social, no como un afuera, sino como constitutivo de los sujetos en posición de estudiantes?; ¿contribuyen las prácticas docentes a problematizar la realidad?; ¿en qué medida las prácticas docentes contribuyen a reproducir lo social y en qué medida contribuyen a dislocar miradas y actitudes reproductoras?; ¿en qué medida en las prácticas docentes se tiene en cuenta la necesidad de contribuir en forma creativa y crítica a la construcción del mercado de trabajo?; ¿cómo pensar una propuesta curricular que tienda puentes entre los conocimientos disciplinares y el contexto geopolítico, económico, social y simbólico que nos constituye y configura el ejercicio de la profesión?; ¿qué acciones institucionales de articulación es necesario encarar?; ¿qué prácticas académicas es necesario poner en valor y cuáles desnaturalizar y transformar?

La formulación de interrogantes no impide trazar un camino para pensar estrategias de acompañamiento de las trayectorias de los estudiantes. Podríamos decir más bien que esos interrogantes constituyen el horizonte desde el cual repensar colectivamente las prácticas. Con respecto a lo que expresan los estudiantes, parece necesario encarar acciones institucionales tendientes a lograr acuerdos que posibiliten sostener un discurso colectivo acerca de que la formación profesional involucra tanto la teoría como la práctica y no termina nunca, pues sostener lo contrario supondría que tanto el conocimiento científico como la sociedad han llegado a su fin.

\section{REFLEXIONES FINALES}

Desde la perspectiva de las trayectorias, la educación se concibe como acompañamiento de las historias cotidianas, para "proponer formas de subjetivación colectiva: desde los otros, que, a fin de cuentas, somos nosotros" (NICASTRO Y GRECO, 2012: 16). A las formas de subjetivación individualista dominantes en los $90-\mathrm{y}$ con fuertes líneas de continuidad en la actualidad, determinadas por el dominio del mercado en el ámbito de lo simbólico- es posible y necesario oponer modos de subjetivación colectiva fundados en la plena conciencia de que no es posible la constitución de un yo sin un nosotros, lo que supone, a la vez, la decisión de vivir juntos.

Es precisamente a esa decisión de vivir juntos la que puede y debe contribuir la arquitectura, concebida y ejercida como profesión cuya razón de ser es lo social, en el sentido de que esta viene a resolver espacios para el desarrollo de actividades colectivas, porque ni en la mayor utopía individualista es posible pensar algún espacio absolutamente individual. En los espacios —al igual que en el tiempo y en cualquier actividad humana- se expresa el complejo entramado que supone, primero, hombres en relación con otros hombres en todas sus formas y, luego, la habilidad por parte del arquitecto de leer e interpretar la cultura, para la ideación y constitución de ámbitos de vida.

La Ley de Educación Nacional 26206 promulgada en 2006 establece la inclusión como principio político y ético de la educación en el país. Esta ley forma parte de un conjunto de leyes tendientes a la ampliación de derechos de personas pertenecientes a sectores o grupos subalternos, promulgadas en los últimos doce años. La universidad, como institución productora de conocimientos y formadora de profesionales, no puede sino hacerse eco de un principio que, indudablemente, tiende a la justicia.

Hoy, los saberes circulan de manera tan fluida que resulta una necedad pensar que habitan en las 
instituciones de educación formal. Sin embargo, la transmisión de esos saberes y del conocimiento científico sigue siendo un desafío tanto para los docentes como para los estudiantes. Obviamente, el desarrollo vertiginoso de la ciencia es uno de los principales factores que inciden en ese desafío. El otro factor - en particular en nuestro países el reconocimiento de la diversidad, puesta hoy en valor como riqueza y no como obstáculo o dificultad. De aquí la necesidad de asumir la educación en su dimensión ético-política, es decir, como tarea que obliga al docente a realizar el máximo esfuerzo por garantizar el acceso de todos los estudiantes al conocimiento - derecho humano inalienable-, y que las decisiones curriculares que toma cotidianamente pueden servir tanto a la democratización de la sociedad como a la exclusión de quienes pasan por las instituciones educativas en condiciones de desventaja.

Desde la perspectiva de las pedagogías críticas -y en oposición a la tradición en educación-, el mayor peso de la tarea está puesto en la enseñanza. Ello no quiere decir, sin embargo, que la cuestión se resuelva en términos de la utilización de adecuadas técnicas de transmisión del conocimiento. Significa más bien que es necesario repensar el posicionamiento del docente, no solo con respecto a los logros de los estudiantes, sino con respecto a la representación que de estos y de la tarea de educar tienen. Para no despojar la educación de su sentido político, es necesario reconocer que los estudiantes ingresan en las aulas "en condiciones que no son definitivas ni inexorables" (SOUTHWELL, 2009), aunque sea necesario conocerlas y ponerlas en valor.

Pero educar no es tarea fácil. Como plantea TERIGI (2013), el trabajo docente es una actividad colectiva y transformadora, y se lo considera tensionado en una suerte de paradoja en la cual, al mismo tiempo que se lo carga de críticas, se espera que realice un aporte crucial para la mejora de la educación. "Comprender esta paradoja es imprescindible para entender algunos de los dilemas actuales del trabajo docente" (NOVOA, 1999. Citado por TERIGI, FLAVIA, en Los saberes docentes. Formación, elaboración en la experiencia e investigación), actividad que merece se subraye el contexto - sea este institucional o social amplio-y se considere que "La peculiar relación de los docentes con el saber tiene efectos en su posición epistémica y sobre su autoridad social" (IBÍDEM, p. 13).

En consonancia con el planteo de MASSCHELEIN acerca de la importancia de dislocar la mirada, PHILIPPE MEIRIEU (2013) se pregunta qué transmitir a las nuevas generaciones y cómo pensar una transmisión emancipadora y no reproductora. Según MEIRIEU es necesario que los docentes acudan a aquellos saberes que, por un lado, permiten la integración de los estudiantes a la sociedad y, por otro, propicien la ruptura con posturas impuestas o heredadas, mediante la búsqueda de un pensamiento autónomo que les permita construir una mirada crítica. En consonancia con estos saberes, los docentes además deberán orientar la enseñanza hacia conocimientos que faciliten hacerse cuestionamientos antropológicos, y con ello, reconocerse a sí mismos y en su relación con los demás. Ello también supone, en términos institucionales, "un nuevo y necesario 'contrato social' entre Universidad y Sociedad" (CARRIZO, 2004: 1), sobre todo considerando que asistimos a procesos de profundas transformaciones en todos los ámbitos, que se traducen en una reedición y profundización del debate acerca de la necesidad de "cimentar una nueva visión de la educación superior" (ÍDEM).

Asumir la educación como derecho social es ponerse en el orden de la utopía, lo que obliga a reconocer que, para que se produzca el 
aprendizaje, no alcanza con la mera presencia de profesores y estudiantes en un espacio formalmente "destinado" a la educación. Para que se produzca es necesario acompañar a los estudiantes, pensados ya no como receptáculos de conocimientos ni como "esponjas" capaces de apropiarse de todo lo que se les ofrece, sino como sujetos diversos, constituidos en y por una historia social que pesa también en la constitución de los profesores como profesionales.

\section{REFERENCIAS BIBLIOGRÁFICAS}

CARRIZO, Luis (2004) Producción de conocimiento y políticas púbicas. Desafíos de la Universidad para la gobernanza democrática. Universidad Autónoma Metropolitana, Xochimilco, Méjico. Agosto de 2004.

FERRY, Giles (1997) Pedagogía de la Formación. Bs. As.: Ediciones Novedades Educativas.

FOGAR, Mariela y SILVA, Carlos. Los estudiantes y su formación. Escenarios y tensiones en la educación técnica. Estudio de casos del Profesorado en Tecnología del INTET y P. Revista Theomai. Estudios Críticos sobre Sociedad y Desarrollo. N. ${ }^{\circ} 30$, Conflictos y problemáticas sociales, segundo semestre de 2014, pp. 172-180.

MASSCHELEIN, Jan (2006). Educar la mirada. La necesidad de una pedagogía pobre. Buenos Aires: Manantial.

MEIRIEU, Philippe (2013) La opción de educar y la responsabilidad pedagógica. Conferencia de Prensa, Buenos Aires, 30 de octubre de 2013.

NICASTRO, S. y GRECO, M. B. (2012) Entre trayectorias. Escenas y pensamientos en espacios de formación. Rosario: Homo Sapiens.

PIAGET, Jean e INHELDER, Bärbel (1975) Psicología del niño. Madrid: Morata.

SOUTHWELL, Myriam. Docentes: la tarea de cruzar fronteras y tender puentes (UNLP / CONICET / FLACSO), Ministerio de Educación de la Nación, Programa de Capacitación Multimedial. (s/f)

SOUTHWELL, Myriam (2009) Docencia, tradiciones y nuevos desafíos en el escenario contemporáneo, La formación docente. Complejidad y ausencias. Córdoba: Encuentro Grupo Editor.

TERIGI, Flavia (2012) Documento básico. Los saberes docentes. Formación, elaboración en la experiencia e investigación. Buenos Aires: Santillana. 